\title{
Postsynaptic Membrane Addition Depends on the Discs-Large-Interacting t-SNARE Gtaxin
}

\author{
David Gorczyca, ${ }^{1 \star}$ James Ashley, ${ }^{1 \star}$ Sean Speese, ${ }^{1}$ Norberto Gherbesi, ${ }^{1}$ Ulrich Thomas, ${ }^{2}$ Eckart Gundelfinger, ${ }^{2}$ \\ L. Sian Gramates, ${ }^{3}$ and Vivian Budnik ${ }^{1}$ \\ ${ }^{1}$ Department of Neurobiology, University of Massachusetts Medical School, Worcester, Massachusetts 01605, ${ }^{2}$ Leibniz Institute for Neurobiology, 39118 \\ Magdeburg, Germany, and ${ }^{3}$ Molecular and Cellular Biology Graduate Program, University of Massachusetts at Amherst, Amherst, Massachusetts 01003
}

\begin{abstract}
Targeted membrane addition is a hallmark of many cellular functions. In the nervous system, modification of synaptic membrane size has a major impact on synaptic function. However, because of the complex shape of neurons and the need to target membrane addition to very small and polarized synaptic compartments, this process is poorly understood. Here, we show that Gtaxin (GTX), a Drosophilat-SNARE (target-soluble $\mathrm{N}$-ethylmaleimide-sensitive factor attachment protein receptor), is required for expansion of postsynaptic membranes during new synapse formation. Mutations in $g t x$ lead to drastic reductions in postsynaptic membrane surface, whereas gtx upregulation results in the formation of complex membrane structures at ectopic sites. Postsynaptic GTX activity depends on its direct interaction with Discs-Large (DLG), a multidomain scaffolding protein of the PSD-95 (postsynaptic density protein-95) family with key roles in cell polarity and formation of cellular junctions as well as synaptic protein anchoring and trafficking. We show that DLG selectively determines the postsynaptic distribution of GTX to type I, but not to type II or type III boutons on the same cell, thereby defining sites of membrane addition to this unique set of glutamatergic synapses. We provide a mechanistic explanation for selective targeted membrane expansion at specific synaptic junctions.
\end{abstract}

Key words: postsynaptic plasticity; neuromuscular junction; Discs-large; Drosophila; membrane addition; MAGUK

\section{Introduction}

Targeted membrane addition is of fundamental importance to the development, function, and plasticity of neuronal synapses, during which profound and coordinated structural alterations of both the presynaptic and postsynaptic membrane compartments must occur (Lahey et al., 1994; Marrs et al., 2001; Matsuzaki et al., 2004; Toresson and Grant, 2005; Lee et al., 2006). Despite its importance, this process is poorly understood in the nervous system (Horton and Ehlers, 2004). The Drosophila larval neuromuscular junction (NMJ) is an excellent model system to study synaptic membrane addition, because the area and complexity of the postsynaptic membrane increases drastically during larval development. Over the course of $4 \mathrm{~d}$, a massive amount of membrane is added to a very small postsynaptic junction comprising $<1 \%$ of the total muscle surface area. This process results in the

\footnotetext{
Received July 24, 2006; revised Nov. 20, 2006; accepted Dec. 20, 2006.

This work was supported by a Max Planck Award for international collaborations from the Alexander von Humboldt Society and the Max Planck Society (E.D.G.) and by National Institutes of Health Grant R01 NS042629 (V.B.). We thank Drs. Mary Munson and Michael Gorczyca for helpful comments on this manuscript and Drs. Alex Keene Tom Schwarz, Hermona Soreq, and Noam Ziv for helpful discussions. We also thank Dr. Karl Heinz Smalla for his advice with the biochemistry, Dr. Greg Hendricks (University of Massachusetts Medical School Electron Microscopy Facility), and Brian Schneider for help with the Volocity software.

*D.G. and J.A. contributed equally to this work.

Correspondence should be addressed to Vivian Budnik, Department of Neurobiology, University of Massachusetts Medical School, 364 Plantation Street, Worcester, MA 01605. E-mail: Vivian.Budnik@umassmed.edu.

D. Gorczyca's present address: Program in Neuroscience, University of California, San Francisco, San Francisco, CA 94143.

L. S. Gramates's present address: Department of Cellular and Molecular Biology, Harvard University, Cambridge, MA 02138.

DOI:10.1523/JNEUROSCI.3160-06.2007

Copyright $\odot 2007$ Society for Neuroscience $\quad 0270-6474 / 07 / 271033-12 \$ 15.00 / 0$
}

formation of a highly convoluted and multilayered postsynaptic membrane specialization [the subsynaptic reticulum (SSR)] where receptors, cell adhesion molecules (CAMs), and ion channels are anchored, and which also contributes to local translation of postsynaptic proteins.

Membrane associated guanylate kinases (MAGUKs) such as Drosophila Discs-Large (DLG) and its mammalian relative postsynaptic density protein-95 (PSD-95) have been implicated in controlling the size, shape, and function of synaptic structures. Mutations in $d l g$ lead to striking defects in SSR expansion (Lahey et al., 1994), and modified levels of PSD-95 are associated with changes in the number and size of dendritic spines (El-Husseini et al., 2000a). Most hypotheses regarding the function of MAGUKs during synaptic assembly and function have centered around their role in clustering ion channels, transduction of calcium signals, and linkage of these proteins to the plasma membrane and cytoskeleton (Tejedor et al., 1997; Thomas et al., 1997; Zito et al., 1997; Nourry et al., 2003). MAGUKs, however, may also contribute to synaptic structure and function by regulating membrane addition. An association of synaptic MAGUKs with membranous compartments is widely recognized (El-Husseini et al., 2000b; Thomas et al., 2000; Sans et al., 2001), and PSD-95 and its paralog SAP102 (synapse-associated protein 102) associate with the exocyst complex protein Sec8 in neurons (Riefler et al., 2003).

Within the SSR, DLG is required for clustering Shaker potassium channels (Sh) and the CAM Fasciclin II (FasII), as well as for SSR expansion (Lahey et al., 1994; Budnik et al., 1996; Guan et al., 1996; Tejedor et al., 1997; Thomas et al., 1997; Zito et al., 1997). Strikingly, however, Sh and fasII mutations do not affect the SSR 
(Tejedor et al., 1997; Thomas et al., 1997). This implies that DLG plays a role in the structure and regulation of postsynaptic membranes independent of its interaction with Sh or FasII.

Addition of membranes by vesicle fusion commonly involves soluble $\mathrm{N}$-ethylmalemide-sensitive factor attachment protein receptor (SNARE) proteins as the minimal fusion machinery (Sollner et al., 1993). Here, we report the isolation of a postsynaptic DLG-interacting target-SNARE ( $\mathrm{t}$-SNARE), guanylate kinaselike (GUK)-interacting syntaxin [Gtaxin (GTX)], that is involved in this process. GTX shares sequence similarity with vertebrate Syntaxin-18 (Hatsuzawa et al., 2000) and yeast Ufelp (Lewis and Pelham, 1996), the latter of which can mediate homotypic endoplasmic reticulum (ER) membrane fusion in the absence of any other known SNARE (Patel et al., 1998). We show that GTX and DLG are physically linked, that this interaction is required for postsynaptic localization of GTX, and that DLG regulates the formation of SDS-resistant SNARE complexes containing GTX. Mutations in $g t x$ phenocopy the reduced SSR and bouton number in $d l g$ mutants, and GTX overexpression results in ectopic formation of SSR-like structures. We propose that GTX is a major effector for DLG-dependent addition of postsynaptic membranes during synapse development.

\section{Materials and Methods}

Fly strains. All fly stocks were maintained on standard Drosophila food. The following fly strains were used: $d l g^{X I-2}$ and $d l g^{v 59}$ (Woods and Bryant, 1989), Df(1)N71 (a deficiency in the dlg locus), Df(3R)Exel 7357 [a deficiency of the $g t x$ locus deleting 96A2-96A13; referred to as $\operatorname{Df}(g t x)$; Bloomington Stock Center (BSC), Bloomington, IN], the Gal4 strains BG487 and C57 (Budnik et al., 1996), the Gal4 strain T80 (Rorth, 1996), and the upstream activator sequence (UAS) stock UAS-mCD8-GFP (BSC). The $g t x^{e x 6}$ mutant was generated through excision of the EPgy2 P-element insertion, $42 \mathrm{bp}$ from the start codon within the first exon of GTX, in the EY08095 line (BSC). Because $g t x^{e x 6}$ mutant larvae were very weak, they were transferred from the vials to $10 \mathrm{~cm}$ Petri dishes containing enriched larval medium [ $1 \%$ agar, $5 \%$ sucrose, $2.5 \%$ yeast extract, $2 \%$ inactive yeast, $0.5 \%$ propionic-phosphoric acid (9:1)] and transferred to fresh plates every day. The UAS-GTX stock was generated by cloning a full-length $g t x \mathrm{cDNA}$ into the pUAST vector and introducing it to flies by germ-line transformation.

Yeast two-hybrid assay. The yeast two-hybrid screen, using the GUK domain of DLG as a bait was described by Mathew et al. (2002). Briefly, the GUK domain of DLG (amino acids 765-961) was cloned into the pLexA (Clontech, Cambridge, UK) vector and used as a bait to screen a late embryonic cDNA library cloned into pB42AD (Clontech). Positive interactions were determined by expression of the reporter genes $\beta$-galactosidase ( $\beta$-gal) and leucine (LEU). Additional characterization of the interactions was performed using full-length GTX clones and a deletion series of the DLG GUK domain also described by Mathew et al. (2002). The GTX gene was identified by blasting positive yeast twohybrid clones against the Drosophila genome using blast from flybase. The resulting gene was Syntaxin-18, identified as CG13626, with GenBank number AAF56317.

Generation of anti-Gtaxin antibodies. Full-length GTX and a truncated form of GTX (amino acids 11-395) were cloned into the pET (Novagen, Madison, WI) expression vector, and His-tagged antigen was generated in bacteria and purified on a nickel column (Novagen). The purified antigen was injected into rabbits for antisera collection. The resulting antisera was further affinity purified on a GST column. Briefly, fulllength GTX was cloned into the pGEX expression vector for bacterial production of GST-GTX fusion protein, and the protein was incubated with a glutathione-agarose column. The GST-GTX was then crosslinked to the column, washed, and then incubated with the serum. The antibody was eluted from the column using a glycine- $\mathrm{HCl}$ solution, and then, using a buffer exchange column, PBS was exchanged for the glycine-HCl.

Immunocytochemistry and morphometric analysis of NMJs. For immunocytochemistry, larvae were dissected under calcium-free saline and fixed using either 4\% paraformaldehyde or Bouin's fixative. Samples were then washed in phosphate buffered Triton X-100 and incubated with antibodies overnight. Antibody concentrations were as follows:

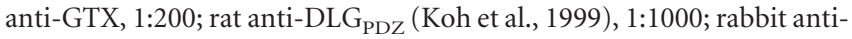
DLG $_{\text {PDZ }}$ (Koh et al., 1999), 1:20,000 (for NMJ staining), and 1:10,000 (for DLG muscle network staining); anti-Spectrin, 1:30 (3A9; Developmental Studies Hybridoma Bank, University of Iowa, Iowa City, IA); 1:1000 anti-Scrib (Roche et al., 2002), 1:200 anti-HRP, 1:10 anti-GluRIIA (8B4D2; Developmental Studies Hybridoma Bank) (Ruiz-Canada et al., 2004). FITC-, Texas Red-, and Cy5-conjugated secondary antibodies used were obtained from Jackson ImmunoResearch (West Grove, PA). Samples were imaged using a Zeiss (Oberkochen, Germany) Pascal Confocal Microscope using a $63 \times(1.4$ numerical aperture) objective and analyzed using Zeiss LSM software. Comparison between different genotypes was done by performing the stainings simultaneously in all of the genotypes and by imaging them with identical nonsaturating confocal parameters.

Analysis of GTX and DLG intensities were performed using the ImageJ software. Briefly, Type $1 \mathrm{~b}$ boutons were traced to outline the region of interest using maximum intensity pixel projection of single arbors, and average fluorescence intensities within the regions of interest were obtained. Analysis of GTX puncta in the muscle was also done with ImageJ. By choosing a region in muscle 6 near the site of innervation, puncta were quantified based on size and intensity (via thresholding). Background values were found to be equal between controls and wild type; therefore, the same threshold parameters were applied to both. Once puncta were segmented, measurements were run for average intensity and average size $\left(\mu \mathrm{m}^{2}\right)$. Statistical analysis was performed using the Student's $t$ test.

Three-dimensional image analysis was performed using Volocity 4.0 software (Improvision, Lexington, MA), using image stacks collected from the Zeiss Pascal Confocal. For measurements of the volume ( $V$ ) occupied by Spectrin in the postsynaptic area, single terminal boutons were selected and analyzed as three-dimensional volumes in Volocity. Presynaptic Spectrin staining was excluded by subtracting the volume occupied by the anti-HRP label (presynaptic bouton volume). The volume occupied by postsynaptic Spectrin staining was expressed as the ratio $V_{\text {post-Spectrin }} / V_{\text {bouton }}$. This ratio was normalized to wild-type controls dissected and processed for immunocytochemistry simultaneously.

To quantify bouton number, early third instar larvae were size matched to gtx $x^{e x \sigma}$ mutants, labeled with anti-HRP, and the total number of synaptic boutons on muscles 6 and 7 were counted in segment A3. To control for possible developmental differences, the length and width of each muscle was measured and used to normalize bouton number (Ashley et al., 2005). Statistical significance was determined using Student's $t$ test. Samples were prepared for transmission electron microscopy as described previously (Packard et al., 2002). Analysis of SSR length was done as described by Budnik et al. (1996).

Western blots and immunoprecipitations. For both Western blots and immunoprecipitations, samples were dissected under ice-cold calciumfree saline. For Western blots, the tissue was homogenized and used immediately after dissection. All steps were done at $4^{\circ} \mathrm{C}$. Eight samples were dissected for each genotype and homogenized at $4^{\circ} \mathrm{C}$ in RIPA (radioimmunoprecipitation assay) buffer containing protease inhibitors. Homogenates were spun at $3000 \mathrm{rpm}$, and 2\% SDS loading buffer was added to the supernatants. Each homogenate was split and the aliquots incubated at $42^{\circ} \mathrm{C}$ or $105^{\circ} \mathrm{C}$ heat block, for times described in this paper. For immunoprecipitations, 30 larvae of each genotype were dissected and frozen. The larvae were then homogenized and spun as described above. Supernatants were precleared with either Protein A (Sigma, St. Louis, MO) or Protein A/G (Santa Cruz Biotechnology, Santa Cruz, CA) beads, and the precleared lysate incubated for $4 \mathrm{~h}$ with Protein A, or Protein A/G beads previously bound to either rabbit anti-GTX or rat anti-DLG, respectively. After washing the beads, loading buffer was added, and the solution was heated to $42^{\circ} \mathrm{C}$ for $5 \mathrm{~min}$, run in $8 \%$ SDSPAGE, and transferred onto a nitrocellulose membrane. To probe the blots, the following antibodies were used: anti-GTX (1:5000), rat antiDLG (1:5000) (Koh et al., 1999), or mouse anti-DLG (1:2000, Developmental Studies Hybridoma Bank), anti-Tubulin (1:4000; Sigma), mouse anti- $\alpha$ Spectrin (3A9, 1:1000; Developmental Studies Hybridoma Bank), 
A

B

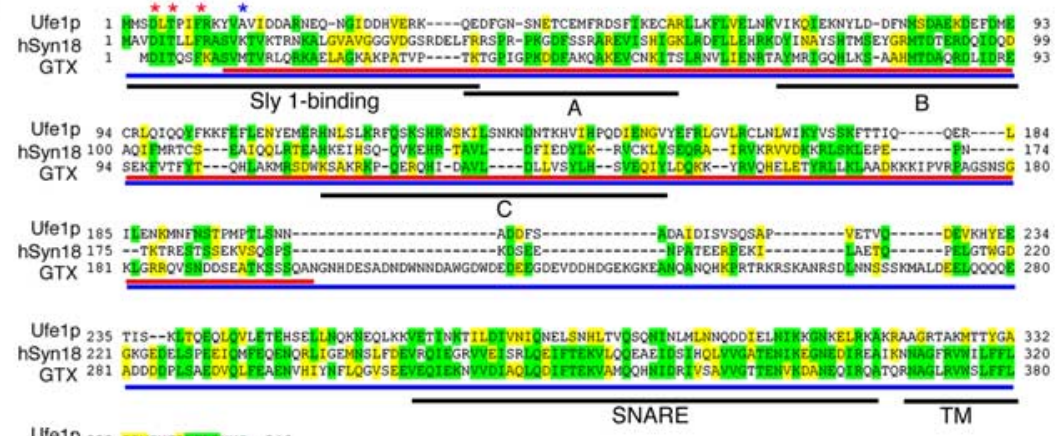

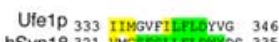

hSyn18 321 whos

TM

wild type

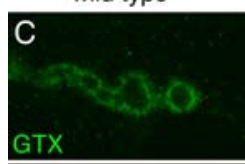

wild type
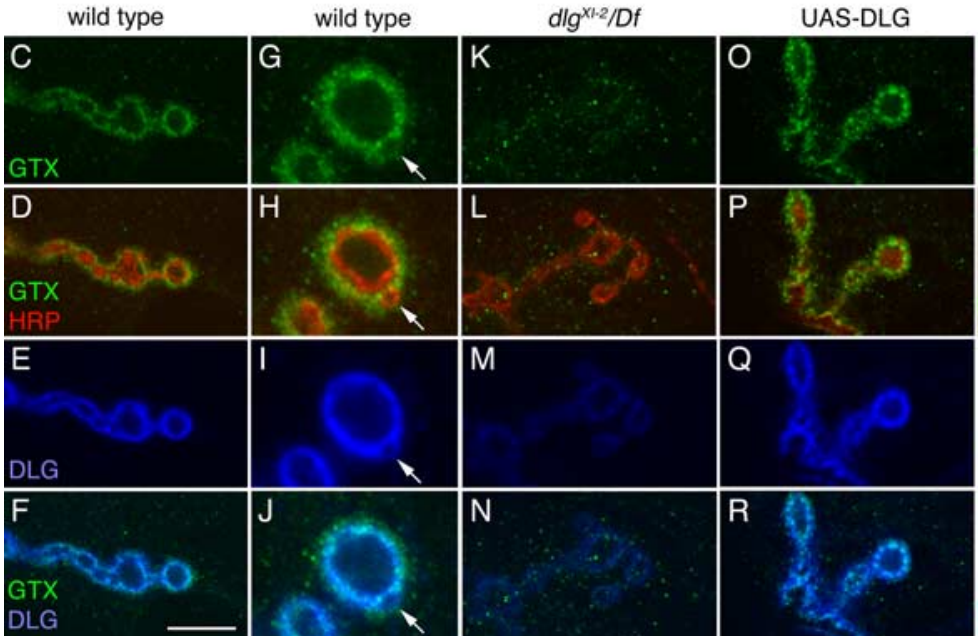

Figure 1. GTX protein structure and synaptic localization. $A$, Organization of GTX into protein domains. (N, N-terminal homologous Sly1 binding site; $\mathrm{A}, \mathrm{B}, \mathrm{C}$, predicted $\mathrm{Ha}$, Hb, and Hc domains; SNARE, coiled-coil SNARE domain; TM, transmembrane domain). B, Protein alignment of yeast Ufe1p, human Syntaxin 18, and GTX. Asterisks represent amino acids required for Sly1 binding (red, conserved; blue, not conserved). Regions of identity are highlighted in yellow, whereas conserved regions are marked in green. Black underlining indicates different protein domains. Red and blue underlining shows the GTX protein regions used to generate the anti-GTX antisera. $\mathbf{C}-\boldsymbol{V}$, Single confocal slices of third instar larval NMJs labeled with anti-GTX (green), anti-HRP (red), and anti-DLG (blue), shown at low $(\boldsymbol{C}-\boldsymbol{F}, \boldsymbol{K}-\boldsymbol{R})$ and high $(\boldsymbol{G}-\boldsymbol{J}, \mathbf{S}-\boldsymbol{V})$ magnification in wild type $(\boldsymbol{C}-\boldsymbol{J}), d l g^{X I-2} / \mathrm{Df}(\mathrm{dlg})(\boldsymbol{K}-\boldsymbol{N})$, and UAS-DLG $(\boldsymbol{O}-\boldsymbol{V})$ overexpressed using the BG487 Gal4. The arrow in $\mathbf{G}-\boldsymbol{J}$ points to a budding bouton. Arrows in $S-V$ point to accumulations of $D L G$, which are accompanied by accumulations of GTX. Scale bar: C-F, K-N, O-R, $7 \mu \mathrm{m} ; \mathbf{G}-J, S-V, 3 \mu \mathrm{m}$.

and anti-Syntaxin1A (8C3, 1:100; Developmental Studies Hybridoma Bank). Quantification of Western blots to document the variation of the $70 \mathrm{kDa}$ band was performed by using ImageJ software and calculating the mean intensity of each $70 \mathrm{kDa}$ band. The mean intensity of each band was then normalized to the loading control mean intensity and expressed as a percentage of wild type.

Electrophysiology. Voltage recordings were performed as in Ashley et al. (2005). Recordings were performed on size-matched larvae, and only those with resting potentials of $-60 \mathrm{mV}$ to $-63 \mathrm{mV}$ were analyzed. Resistance and capacitance were calculated by injecting a $1 \mathrm{nA}$ pulse and recording the steady-state voltage change and time constant of the initial capacitive surge. To determine specific resistance and capacitance, larvae were fixed, and the total surface of each muscle was determined by measuring their length, width, and cross-sectional perimeter using the confocal microscope. No differences in the specific resistance or capacitance were found in any of the genotypes tested. Two-electrode voltage clamp was performed at a holding potential of $-80 \mathrm{mV}$ as described by Budnik et al. (1996) using an Axoclamp2A (Molecular Devices, Union City, CA). Recordings were from muscle 6 segment A3, of size matched early third instar larvae, which were dissected in HL-3 saline containing $0.3 \mathrm{~mm}$
UAS-DLG
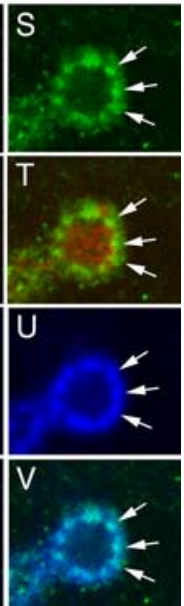

$\mathrm{Ca}^{2+}$ (Stewart et al., 1994) and recorded in HL-3 saline containing $0.5 \mathrm{~mm} \mathrm{Ca}^{2+}$. Recordings were filtered at $1 \mathrm{kHz}$ and digitized using an Instrutech (Port Washington, NY) ITC-16 computer interface. Data were processed using Pulse software (HEKA Electronik, Lambrecht/ Pfalz, Germany) and measured using Mini Analysis software (Synaptosoft, Decatur, GA.). Statistical analysis throughout this study was performed using the Student's $t$ test with unequal variance.

\section{Results}

\section{DLG is required for proper synaptic} localization of GTX

As one of the prototypical MAGUKs, DLG is composed of three PDZ (PSD-95/DiscsLarge/zona occludens-1) domains, an SH3 (Src-homology 3) domain and a catalytically inactive GUK domain. At the NMJ, the lack of an intact GUK domain in $d \lg ^{v 59}$ and $d \lg ^{X I-12}$ mutants is associated with reduced SSR expansion (Lahey et al., 1994; Thomas et al., 1997; Mendoza et al., 2003). To address the function of the GUK domain, we used it as a bait in a yeast twohybrid screen of a late embryonic Drosophila cDNA library. Aside from the previously described protein GUK-holder (Mathew et al., 2002), another protein, GTX, was identified in this screen. The conceptual GTX protein is comprised of 395 amino acids and shares striking sequence identity $(32.4 \%, 15.4 \%)$ and similarity $(46.3 \%, 27.3 \%)$ with human Syntaxin-18 and yeast Ufe1p, respectively (Fig. 1A,B). Like other Syntaxins, GTX contains an N-terminal region similar to the Sly1-binding site (Hong, 2005), a putative central Habc domain, a SNARE domain, and a single-pass transmembrane domain (Fig. 1A).

To assess a possible association of GTX with DLG at synapses, we used antisera generated against either full-length or a segment of GTX. Antisera specificity was confirmed by a dramatic decrease of immunoreactivity at the $\mathrm{NMJ}$ and in Western blots of $g t x$ mutants, and an increase when overexpressed.

GTX was present at NMJs and colocalized with DLG, as expected if both proteins interact in vivo (Fig. $1 C-F$ ). Like DLG, GTX was enriched postsynaptically at glutamatergic type I boutons (Fig. 1G-J) but absent from type II [octopamine-containing (Monastirioti et al., 1995)] and type III [peptide-containing in muscle 12 (Gorczyca et al., 1993)] boutons (data not shown). Like DLG, GTX immunoreactivity was enriched at type Ib boutons, less prominent at type Is boutons (data not shown), and decreased at sites of bouton budding (Fig. 1G-J, arrow). A closer inspection, however, revealed some notable differences in the pattern of GTX and DLG staining. Anti-GTX and anti-HRP (neuronal surface marker) immunoreactivity revealed no colocalization, suggesting that unlike DLG, GTX is absent from the presynaptic membrane (Fig. $1 \mathrm{H}$ ). Postsynaptically, GTX immu- 
noreactivity appeared to spread slightly beyond DLG into the muscle (Fig. $1 J$ ) and appeared punctate compared with DLG.

GTX was also present in puncta throughout the muscle cortical (below the plasma membrane) and subcortical (below the muscle nuclei) regions (Fig. $2 A-$ $E, M-O)$, and these puncta partially colocalized with a dim DLG-immunoreactive cortical network (Fig. 2G-J,M-O) thought to be involved in DLG transport to the synapse (Thomas et al., 2000) (note that in Fig. 2G-J,M,O, detection of DLG in muscle networks versus synaptic DLG required 10 times higher confocal gain).

We next investigated whether DLG was required for proper localization of GTX. The localization of GTX at NMJs was dramatically reduced in both $d g^{X I-2}$ and $d l^{v 59}$ mutant alleles (Woods et al., 1996; Mendoza et al., 2003) (Fig. $1 K-N$ ) (data not shown), demonstrating that DLG is required for synaptic localization of GTX. This was further supported by the observation that targeted postsynaptic overexpression of DLG at NMJs, induced by the muscle Gal4 driver BG487, resulted in increased GTX immunoreactivity at the peribouton zone (Fig. 1O-R, $S-V$, arrows).

Surprisingly, the above effect was very sensitive to DLG dosage. When DLG expression was further increased using the strong muscle Gal4 driver C57, considerable amounts of DLG accumulated in the cortical network (Fig. 2G-J)(Thomas et al., 2000; Razzaq et al., 2001). Although the abundance of DLG at NMJs remained primarily unaffected (Fig. 2C,D,K), postsynaptic GTX was now clearly decreased (Fig. $2 A, B, K$ ). In parallel, elevated GTX levels became evident as prominent puncta associated with the cortical network (Fig. $2 E, F, L$ ). Thus, increased levels of extrasynaptic DLG are accompanied by an enrichment of GTX in submembrane compartments at the expense of synaptic GTX. We conclude that efficient synaptic trafficking of GTX depends on a well-balanced subcellular distribution of DLG.

We next evaluated the biochemical properties of GTX and its physical association with DLG in vivo. Biochemically, SNAREs are known to form SDS-resistant complexes that dissociate with intense boiling (Otto et al., 1997). Body wall muscle extracts were examined by Western blotting after incubation at $42^{\circ} \mathrm{C}$ and after boiling for 5 and $15 \mathrm{~min}$. A prominent $50 \mathrm{kDa}$ band found in all samples corresponded to monomeric GTX (Fig. 3A, arrow). Without boiling, four additional bands of 55, 70, 90, and $140 \mathrm{kDa}$ were detected by antibodies against GTX (Fig. 3A). These extra bands were completely eliminated by boiling for $15 \mathrm{~min}$ (Fig. 3A). All bands were clearly reduced in extracts from a hypomorphic mutant (see below), confirming that they correspond to higher molecular weight complexes containing GTX, although not necessarily exclusively composed of GTX (Fig. 3B). Interestingly, DLG was required to maintain the $70 \mathrm{kDa}$ complex, because in $d l g^{X 1-2}$ this band was decreased to nearly half of wild-type inten- sity (Fig. $3 A, C, E$ ). Conversely, the $70 \mathrm{kDa}$ band showed a $50 \%$ increase in intensity when DLG was overexpressed (Fig. 3C,E), suggesting that DLG regulates the stability of the $70 \mathrm{kDa}$ GTX complex. The decrease in the intensity of the $70 \mathrm{kDa}$ band was observed even if GTX was overexpressed in a $d l g$ mutant background (Fig. 3E). Thus, like other SNAREs, GTX forms SDSresistant complexes. However, unlike other SNAREs, the formation or stability of at least one of these complexes is regulated by DLG. Monomeric GTX as well as some of the higher molecular weight complexes, including the $70 \mathrm{kDa}$ band, were coprecipitated by antibodies to DLG (Fig. $3 F$ ). In wild type, DLG mostly coprecipitated the high molecular weight GTX complexes (Fig. $3 F$ ), but with GTX overexpression, an interaction between monomeric GTX and DLG was observed. The interactions were specific, because antibodies to DLG failed to coprecipitate the related presynaptic SNARE Syntaxin-1A (Fig. 3F).

\section{Gtaxin is required for NMJ expansion and muscle growth}

The functional significance of GTX was determined in $g t x \mathrm{mu}-$ tants. We obtained a P-element insertion (EY08095; $\left.g t x^{P}\right)$ localized in the first exon of $g t x, 42$ bp upstream of the $g t x$ translation start site. This mutant died during embryonic stages when ho- 

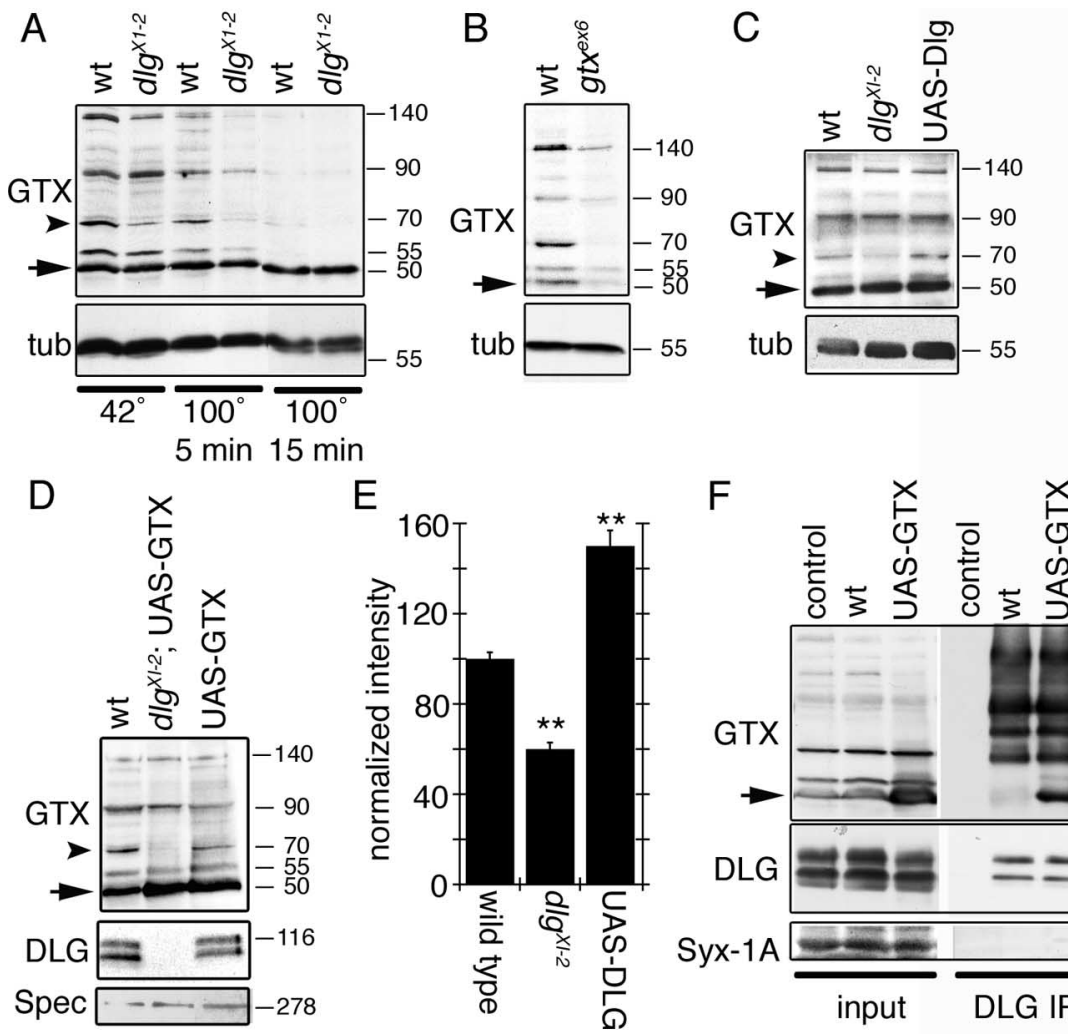

Figure 3. GTX forms SDS-resistant high molecular weight complexes, and DLG regulates these complexes and is associated with GTX in the body wall muscles. $A$, Western blot of wild-type and $d \mathrm{dg}^{X I-2}$ mutant body wall muscle extracts subjected to different temperatures to demonstrate the presence of SDS-resistant high molecular weight complexes and the regulation of some of these complexes by DLG. B, Western blot of wild-type and $g t x^{\mathrm{ex} 6}$ mutant extracts incubated at $42^{\circ} \mathrm{C}$ in loading buffer. $C$, Western blot of body wall muscle extracts from wild type, $d g^{X I-2}$, and larvae overexpressing DLG incubated at $42^{\circ} \mathrm{C}$ in loading buffer. $\mathrm{D}$, Western blot of wild type, overexpression of GTX in a $d l g^{X I-2}$ mutant background, and overexpression of GTX in a wild-type background. $\boldsymbol{E}$, Intensity of the GTX $70 \mathrm{kDa}$ band $(\boldsymbol{A}, \boldsymbol{C}, \boldsymbol{D}$, arrowhead) observed in dlg mutants and after overexpressing DLG, normalized to wild-type intensity. $n=3$ independent Western blots. $\boldsymbol{F}$, Immunoprecipitation of body wall muscle extracts using anti-DLG antibodies. The blots were sequentially probed with antibodies to GTX, DLG, and Syntaxin-1A. Inputs represent 10\% of the extract used for immunoprecipitations. Numbers at the right of the blots represent molecular weights in $\mathrm{kDa}$. The arrow in $A-D$ and $\boldsymbol{F}$ indicates GTX monomer. The arrowhead in $\boldsymbol{A}, \boldsymbol{C}$, and $\boldsymbol{D}$ indicates the $70 \mathrm{kDa}$ GTX complex. tub, Tubulin; Spec, Spectrin; $\mathbb{I P}$, immunoprecipitation.

mozygous and over a small deficiency of the gtx region, $\operatorname{Df}(3 \mathrm{R})$ Exel7357, suggesting that gtx is an essential gene. Mobilization of the P-element yielded additional embryonic lethal lines and a hypomorphic line, gtx $x^{e x 6}$, which survived to the second instar larval stage with a few third instar escapers. In addition, several lines yielded precise excisions, which were perfectly viable. Sequence analysis demonstrated that in $g t x^{e x \sigma}, \sim 500 \mathrm{bp}$ of the original $\mathrm{P}$ element remained within the first exon of gtx, leaving flanking sequences intact.

In $g t x^{e x 6}$ and $g t x^{e x 6} / \mathrm{Df}(g t x)$, GTX immunoreactivity at the NMJ was dramatically decreased, supporting the view that $g t x^{e x 6}$ is a mutation in the gtx gene (Fig. $4 A, B$ ). Synaptic boutons in $g t x^{e x 6}$ mutants were phenotypically very similar to severe $\mathrm{dlg}$ mutants (Thomas et al., 1997; Koh et al., 1999). Like $d l^{X I-2}$ mutants, $g t x^{e x 6}$ mutants displayed enlarged and bulbous boutons, often with only one or two boutons per NMJ branch (Fig. $4 C, D, K, O$ ), along with a severe reduction in the overall number of boutons (Fig. 4S). Muscles in gtx mutants were also significantly smaller than wild type (Fig. 4T), but the number of boutons in $g t x$ mutants remained significantly reduced after normalization to muscle area (Fig. $4 U$ )

We examined the localization of various synaptic proteins that colocalize with GTX at NMJs. A strong reduction of the actin-binding protein Spectrin within the postsynaptic peribouton area was evident (Fig. $4 L, P$ ), whereas the scaffolding and PDZ protein Scribble remained unaffected (data not shown). Although the distribution of DLG at the NMJ was only slightly reduced (Fig. 4M,Q), its distribution within the cortical compartments was altered considerably (see below).

Functional abnormalities at the NMJ of gtx mutants were also observed (Fig. 5). There was a small but significant decrease in the amplitude of excitatory junctional potentials (EJPs), although this phenotype was not rescued by expressing a wild-type gtx transgene ubiquitously (but see below for strong sensitivity of GTX to dosage) (Fig. $5 A, C$ ). Although there was no change in spontaneous miniature EJP (mEJP) amplitude (Fig. 5F), the frequency of mEJPs was decreased, and this phenotype was almost completely rescued by the $g t x$ transgene (Fig. $5 G$ ). Interestingly, the kinetics of the EJP decay was also affected in the mutants, having time constants significantly smaller than wild type, which could be rescued by the gtx transgene (Fig. 5D). These kinetic changes were not caused by a modification in the passive membrane properties of the mutant muscles, because both specific resistance and capacitance were the same as control. To determine whether this change was caused by alteration of glutamate receptor (GluR) properties, muscles were clamped at -80 $\mathrm{mV}$ holding potential to prevent the activation of voltage-gated channels and to examine GluR-mediated currents in isolation. Although a decrease in EJC amplitude was observed, consistent with the EJP measurements (Fig. 5B,E), there was no difference in EJC kinetics (Fig. $5 B, H$ ), suggesting that the change in decay rate observed was not attributable to changes in GluR channel kinetics.

\section{GTX is required for the integrity of the muscle endomembrane system}

DLG is enriched at the SSR but is also associated at low levels with an intracellular membranous network. This network can be labeled with mCD8-GFP, a fusion protein consisting of a transmembrane murine lymphocyte receptor CD8 and GFP, commonly used to label membranes (Fig. 6A1-B3,G1,G2). As shown previously (Thomas et al., 2000), this network is composed of two morphologically different compartments: a cortical network localized above the muscle nuclei and at the same level as the SSR (Fig. 6A1-A3,G1), and a deeper subcortical network positioned underneath the muscle nuclei (Fig. 6B1-B3,G2). The subcortical network is separated by a few micrometers from the cortical network (Fig. 6E1) and intermingles with the myocontractile apparatus. Thus, the subcortical network is likely to represent, at least in part, the sarcoplasmic reticulum and T-tubule network (Razzaq et al., 2001). During early larval stages, DLG is conspicuous in both the cortical and subcortical networks, but as more DLG accumulates at the NMJ in older larvae, its presence in the net- 
work is markedly reduced. DLG traffics from this network to postsynaptic sites, and specific DLG protein domains are involved during different steps of this trafficking (Thomas et al., 2000).

In muscles expressing mCD8-GFP, the cortical membrane compartment appeared as strongly fluorescent patches connected by weakly labeled side arms (Fig. 6A1). DLG was present at low levels in the patches but was enriched in the arms (Fig. 6A2,A3). The subcortical compartment was composed primarily of longitudinal strands, as visualized with mCD8-GFP or anti-DLG antibodies, and both labels partially overlapped (Fig. 6B1$B 3, G 2)$. The cortical and subcortical networks appeared connected by tubules perpendicular to the muscle surface, which were labeled by both mCD8-GFP and anti-DLG (Fig. 6F1,F2).

In $g t x^{e x 6}$ mutants, the configuration of the membranous networks and levels of DLG within the networks were altered (Fig. 6C1-D3,H1,H2). In particular, in the cortical network, the fluorescent mCD8GFP patches were dramatically reduced in size (Fig. 6C1), and DLG accumulated in regions surrounding the residual patches (Fig. $6 C 2, C 3, H 1$ ). The intensity of the mCD8-GFP label at the SSR was also dramatically reduced and failed to completely envelop synaptic boutons (Fig. 6, compare I1-I3 with J1-J3). Additionally, in gtx mutants, the separation of the cortical and subcortical networks was lost (Fig. 6, compare $E 1$ and $K 1$ ). In addition, most of the mCD8-GFP fluorescence accumulated in the subcortical network, and DLG levels were dramatically increased in this network (Fig. 6D1$D 3, H 2)$. Overall, it appeared as if the cortical network had collapsed into the subcortical network in the mutant. Indeed, muscle nuclei, which in wild type are located between the cortical and subcortical networks (Fig. 6E1), appeared flattened in $g t x^{e x 6}$ mutants (Fig. $6 K 1$ ).

\section{The synaptic and muscle GTX functions require different GTX dosage}

To demonstrate the specificity of the phenotypes, we performed rescue experiments by expressing a $g t x$ cDNA (UAS-GTX) in $g t x^{e x 6}$ mutants. Expressing UAS-GTX ubiquitously, using the weak Gal4 driver T80, resulted in complete rescue of phenotypes, including lethality, bouton morphology, and muscle size, demonstrating that the phenotypes were caused by mutations in $g t x$ (Fig. 4S-U). Because GTX is expressed postsynaptically, and because expressing GTX with a presynaptic Gal4, C380 (Budnik et al., 1996), did not rescue the lethality, we next used musclespecific Gal4 drivers. With the strong pan-muscle Gal4, C57 (Budnik et al., 1996), the lethality was rescued as was the muscle size (Fig. 4T). However, no rescue of bouton phenotypes was observed (Fig. 4S, $U$ ).

This result was highly surprising in light of the overall rescue obtained with the ubiquitous T80-Gal4. However, wild-type
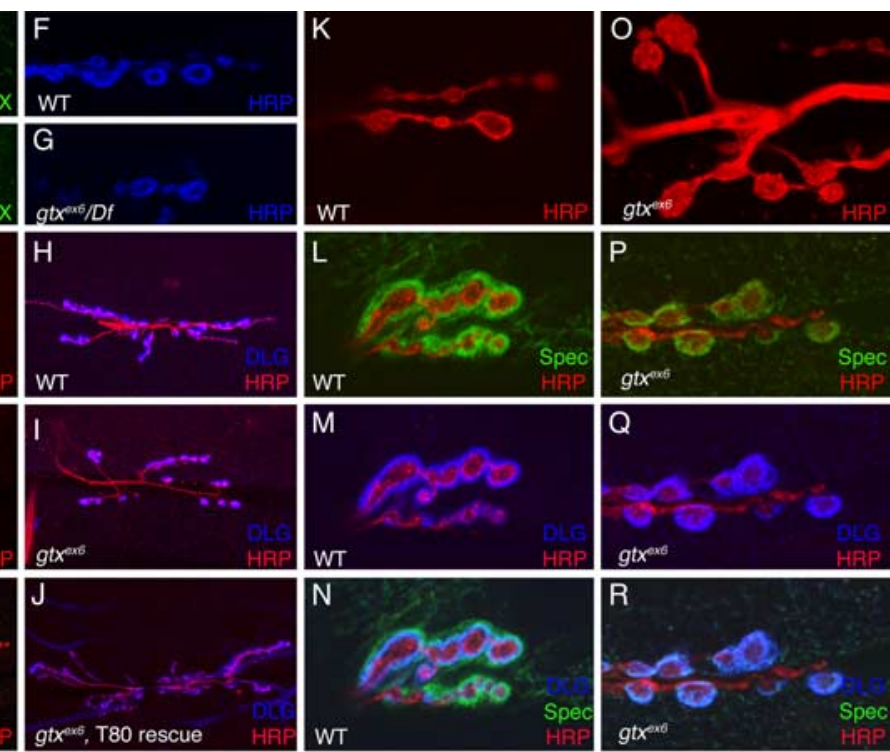

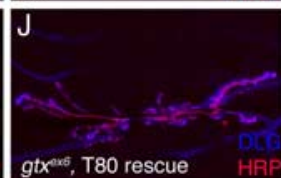

$\mathrm{T}$

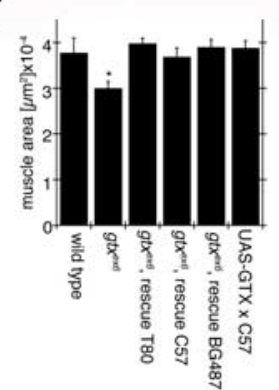

U

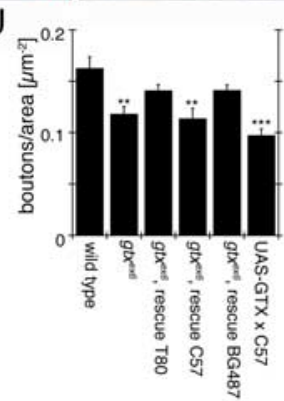

Figure 4. Abnormal NMJs in gtx mutants. $\boldsymbol{A}-\boldsymbol{R}$, Confocal images of third instar larval NMJs stained with anti-GTX (green) and anti-HRP (blue) $(\boldsymbol{A}, \boldsymbol{B}, \boldsymbol{F}, \boldsymbol{G})$, anti-HRP (red) and anti-DLG (blue) $(\boldsymbol{C}-\boldsymbol{E}, \boldsymbol{H}-\boldsymbol{J})$, anti-HRP (red) $(\boldsymbol{K}, \boldsymbol{O})$ and anti-Spectrin (Spec) (green), area $(\boldsymbol{U})\left(n=12\right.$ for each genotype). ${ }^{*} p<0.05 ;{ }^{* *} p<0.001 ;{ }^{* * *} p<0.0001$. Scale bar: $\boldsymbol{A}, \boldsymbol{B}, \boldsymbol{F}, \mathbf{G}, \boldsymbol{K}-\boldsymbol{R}, 7 \mu \mathrm{m} ; \boldsymbol{C}-\boldsymbol{E}, \boldsymbol{H}-\boldsymbol{J}, 35 \mu \mathrm{m}$.

NMJs overexpressing the gtx transgene with the strong C57 muscle driver revealed that GTX accumulated in the form of very large cytoplasmic vesicles (Fig. $7 F-H$ ), which were polarized in their distribution to the synaptic (visceral) side of the muscles (Fig. $7 K$ ). These GTX-positive structures formed in a DLGindependent manner, because they were also observed in a $\mathrm{dlg}$ mutant background (see below). The vesicular nature of these structures was demonstrated by using mCD8-GFP to label membranes, which showed that the structures were bounded by GFP fluorescence (Fig. 7J). In contrast, no GTX was observed at synaptic boutons (Fig. 7I). Additionally, the NMJs of larvae expressing GTX with the strong C57 muscle driver had defects similar to $g t x^{e x 6}$, including an abnormal NMJ morphology (Fig. 7G), and decreased bouton number (Fig. 4S, $U$ ). Thus, overexpressing large amounts of GTX exerts a dominant-negative effect at the NMJ, providing an explanation for the lack of rescue of the NMJ phenotypes in $g t x$ mutants with strong expression of GTX in the muscles. Indeed, examination of larvae in the ubiquitous T80mediated rescue demonstrated that GTX became localized to the $\mathrm{NMJ}$ in these animals (see below). Flies overexpressing GTX with the strong C57 muscle driver were viable and had normal muscle size (Fig. 4S). Thus, the requirement for GTX for survival and muscle growth can be genetically separated from its synaptic role at NMJs by varying the GTX dose within the muscles. 
A

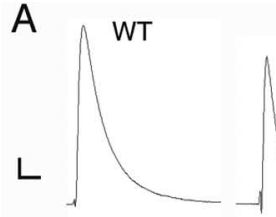

C EJP amplitude

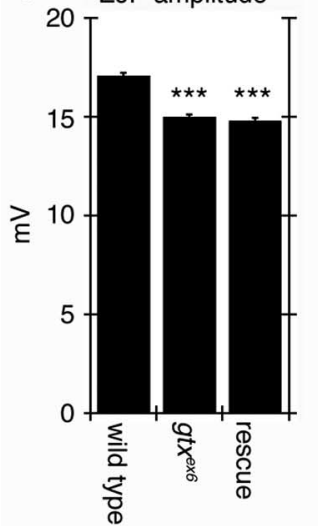

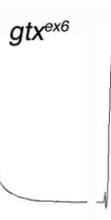

D

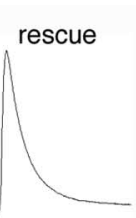

EJP decay

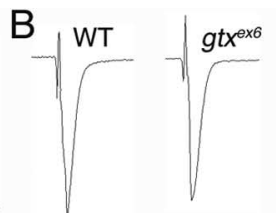

E EJC amplitude

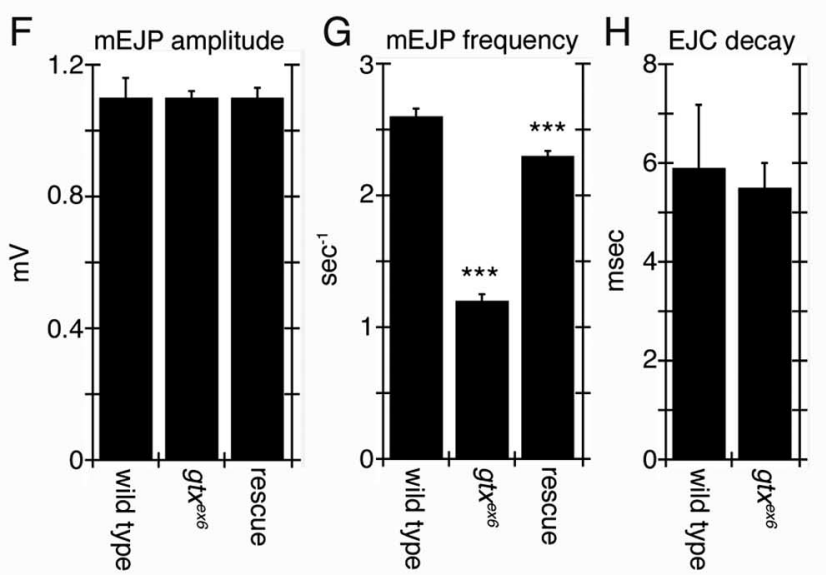

Figure 5. Electrophysiological defects in gtx mutants. $A$, Representative EJP traces of wildtype, $g t x^{e \times 6}$, and $g t x$ transgene expression in a $g t x^{e \times 6}$ mutant background using the T80 Gal4 driver. $\boldsymbol{B}$, Representative voltage-clamp EJC recordings of wild-type and $g t x^{e \times 6}$ mutants. $\boldsymbol{C}-\boldsymbol{H}$, quantification of EJP amplitude $(\boldsymbol{C})$, EJP decay time constant $(\boldsymbol{D})$, EJC amplitude $(\boldsymbol{E})$, mEJP amplitude $(\boldsymbol{F})$, mEJP frequency $(\boldsymbol{G})$, and EJC decay constant $(\boldsymbol{H}) . n=7$ for all genotypes. ${ }^{* *} p<$ 0.001; ${ }^{* *} p<0.0001$. Calibration: $\boldsymbol{A}, 2 \mathrm{mV}, 40 \mathrm{~ms} ; \boldsymbol{B}, 3 \mathrm{nA}, 15 \mathrm{~ms}$.

This dosage effect was further analyzed by using an alternative Gal4 driver, BG487 (Budnik et al., 1996). The pattern of Gal4 expression in BG487 is present in an anteroposterior gradient in muscles 6 and 7, with Gal4 expression being the highest at abdominal segment A1 and rapidly decreasing in more posterior segments. Interestingly, we found that the accumulation of GTX in cytoplasmic vesicles also decreased in an anteroposterior direction (Fig. 7E), whereas, simultaneously, a gradual increase of synaptic GTX was obvious. In abdominal segment A3, GTX was normally localized to synapses, and there was a rescue of the synaptic mutant phenotypes (Fig. $4 S, U$ ). Thus, normal function of GTX at the NMJ is strongly dependent on the dosage of GTX.

\section{GTX is required for proper development of the SSR}

Previous examination of $d l g$ mutants showed that DLG is required for the expansion of the SSR (Lahey et al., 1994; Budnik et al., 1996; Guan et al., 1996). Examination of gtx $x^{\text {ex6 }}$ mutant NMJs at the ultrastructural level revealed an increase in bouton crosssectional area and a decreased SSR length (Fig. $8 A, B, H$ ). These

phenotypes were rescued in segment A3 by expressing GTX in $g t x^{e x 6}$ mutant background using the weak (in segment A3) muscle driver BG487 (Fig. $8 H$ ). Thus, at the NMJ, GTX (like DLG) is required for normal development of the SSR. We found no differences in other parameters, such as the number of active zones (Fig. $8 \mathrm{H}$ ). However, because gtx mutants have larger boutons, the number of active zones normalized by the bouton cross-sectional area was significantly reduced (Fig. $8 \mathrm{H}$ ).

Changes in SSR volume were clearly reflected by changes in the postsynaptic volume occupied by Spectrin. For example, overexpression of DLG in muscles using the BG487 driver results in an expanded SSR (Budnik et al., 1996), and this was reflected in an increased postsynaptic Spectrin volume (Fig. 8I). Similarly, mutations in $d l g$ and in $g t x$ result in decreased SSR (Budnik et al., 1996; our study), and this was paralleled by a decrease in Spectrin volume (Fig. 8I). This correlation between SSR size and Spectrin volume allowed us to look at the SSR in a number of genotypes, without performing electron microscopy. Using this method, we found that overexpressing GTX in wild-type or gtx mutants with the strong muscle C57 driver reduced the SSR volume to an extent similar to gtx and $d l g$ mutants (Fig. 8I). This is consistent with the model that high level overexpression of GTX has a dominant-negative effect on SSR formation. In contrast, overexpressing GTX with the weaker (at segment A3) Gal4 driver BG487 resulted in normal SSR (Fig. 8I). Overexpressing GTX with BG487, however, was not sufficient to rescue the reduced SSR in $d l g$ mutants, consistent with the idea that DLG is required for proper targeting of GTX to postsynaptic sites and thus the expansion of SSR membranes (Fig. 8I). Interestingly, however, overexpressing both DLG and GTX with BG487 decreased the SSR expansion observed by expressing DLG alone (Fig. $8 I$ ). This is likely because of the observations that precise levels of both DLG and GTX are required for proper localization of each protein to the SSR.

Because overexpressing GTX at high levels using C57 gives rise to a dominant-negative phenotype, which phenocopies gtx mutants, and because this manipulation results in the formation of large cytoplasmic structures, we looked at the ultrastructural phenotypes in larvae strongly overexpressing GTX in muscles. Most strikingly, overexpressing GTX with C57 gave rise to highly folded membranous structures or large vesicles in the cytosol (Fig. 8C-G). Also, infoldings of the muscle plasma membrane resembling the SSR, but distant from synaptic regions, could be observed (Fig. 8C,E). The luminal side of the membrane in some of the large cytoplasmic structures was coated with fuzzy electron dense material that resembled the muscle basal lamina (Fig. $8 G$, arrow). Also, the infoldings at extrasynaptic muscle regions contained a similar material that was continuous with the muscle basal lamina (Fig. 8E, arrows). Therefore, ectopic expression of GTX promotes the formation of ectopic SSR-like structures.

The foldings of the membranes in these cytoplasmic and surface SSR-like structures could be observed by confocal microscopy (Fig. 9A). To determine whether these ectopic SSR-like structures depended on the presence of DLG, we examined the effects of overexpressing GTX in a $\mathrm{dlg}^{\mathrm{XI-2}}$ mutant background by confocal microscopy. We found that these structures could also be observed in $d l^{x I-2}$ mutants (Fig. $9 B$ ), suggesting that these were the result of increased GTX levels with independence of DLG.

\section{Discussion}

DLG plays important roles at synapses because of interactions with a variety of binding partners (Tejedor et al., 1997; Thomas et 

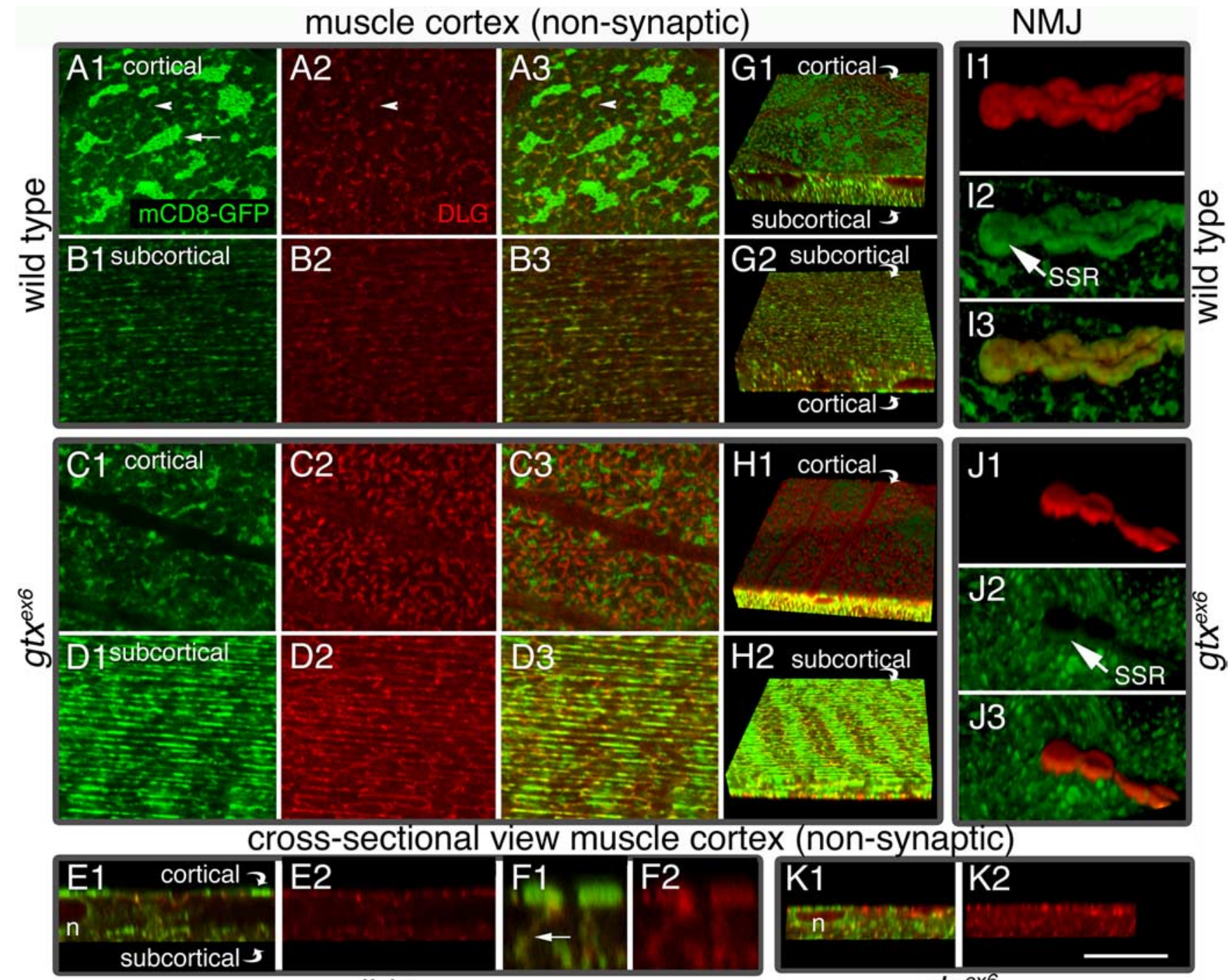

wild type
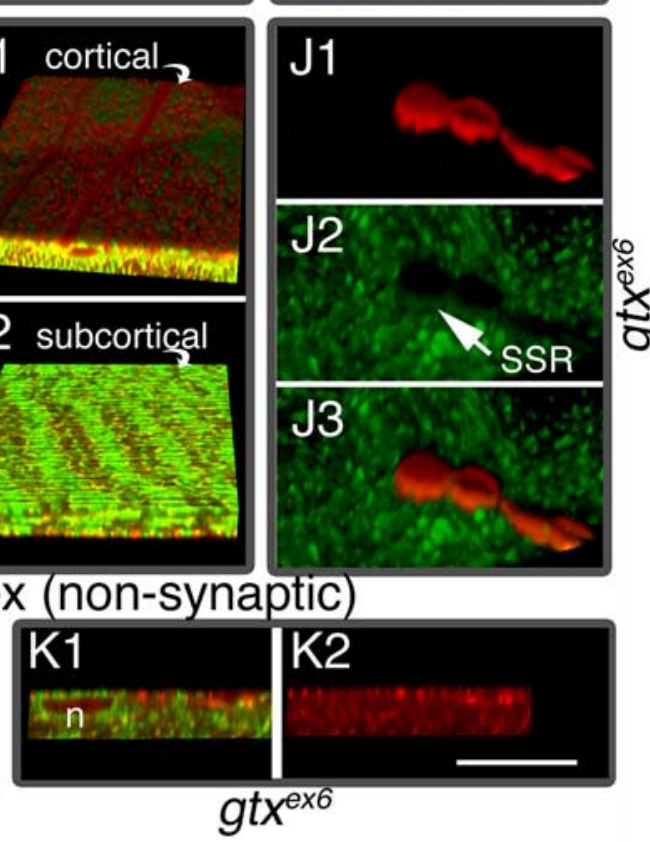

Figure 6. Muscle endomembrane system in wild-type and gtex ${ }^{e x 6}$ larvae. Views of muscle extrasynaptic regions (A1-D3, G1-H2) and of synaptic boutons (II-J3) in preparations labeled with mCD8-GFP (green) and anti-DLG (red) in wild type (A1-B3, E1-G2, I1-I3) and gtxex6 (C1-D3, H1, H2,J1-K2). $\mathbf{A 1}$, Single confocal slices at the muscle cortex, near the muscle surface (A3, C1-C3), and single confocal slices at the subcortical muscle region, below the nuclei (B1-B3, D1-D3). G1, G2, H1, H2, Three-dimensional rendering of confocal slices spanning the muscle cortex and part of the subcortical region shown from the surface of the muscle $(\mathbf{G 1}, \mathbf{H 1})$ and from the subcortical muscle region $(\mathbf{G} \mathbf{2}, \mathbf{H 2})$. E1-F2, $\mathbf{K 1}, \boldsymbol{K 2}$, Transversal section through a confocal stack showing the relationship between the cortical and subcortical networks. n, Nucleus. F1, F2, A high-magnification view of the muscle area between the cortical and subcortical networks, showing structures connecting the two networks. I1-I3,J1-J3, Three-dimensional rendering of an NMJ branch. mCD8-GFP labels muscle membranes including the SSR, and anti-DLG antibodies label mostly the SSR. Cortical, Below plasma membrane; subcortical, below nuclei. Scale bar: A1-D3, $12 \mu \mathrm{m} ; \mathbf{E 1}, \mathbf{E 2}, \mathbf{G 1}-\mathbf{H 2}, \mathbf{K 1}, \mathbf{K 2}, 20 \mu \mathrm{m} ; \mathbf{I 1} \mathbf{I 3}, \mathbf{J 1}-\mathbf{J 3}, 10 \mu \mathrm{m} ; \boldsymbol{F 1}, \boldsymbol{F 2}, 4 \mu \mathrm{m}$.

al., 1997; Zito et al., 1997; Hung and Sheng, 2001; Pak et al., 2001). Although MAGUKs can regulate the size of synaptic structures, suggesting a role in membrane trafficking (Lahey et al., 1994; Budnik et al., 1996; El-Husseini et al., 2000a), the underlying mechanisms have remained elusive. Here, we show that DLG interacts with the t-SNARE GTX, which functions in a DLGdependent manner during postsynaptic membrane expansion and new synaptic bouton formation. We propose that DLG regulates the spatial localization of membrane addition events through the activity of GTX (Fig. 9C,D).

Role of DLG and GTX during synapse development

dlg mutants have diverse synaptic phenotypes (Lahey et al., 1994; Budnik et al., 1996; Thomas et al., 1997), including abnormal NMJs with fewer boutons, increased neurotransmitter release, and a reduced SSR. Some of these arise because of the roles of DLG-binding partners, such as FasII and Sh (Budnik et al., 1990; Schuster et al., 1996a,b; Tejedor et al., 1997; Thomas et al., 1997; Zito et al., 1997). However, the mechanisms by which DLG regulates the size of the SSR have remained elusive.

The SSR is a dynamic membrane system where synaptic proteins localize in a spatially restricted manner (Petersen et al., 1997; Tejedor et al., 1997; Thomas et al., 1997; Sone et al., 2000). The SSR is also a trafficking compartment where regulation of signaling and synaptic protein function occurs through vesicle cycling (Mathew et al., 2003, 2005). At its periphery, the SSR shows association with machinery for local translation of synaptic proteins, potentially constituting an adapted ER-like structure (Sigrist et al., 2000). The complexity of the SSR results from continuous membrane addition throughout larval development 

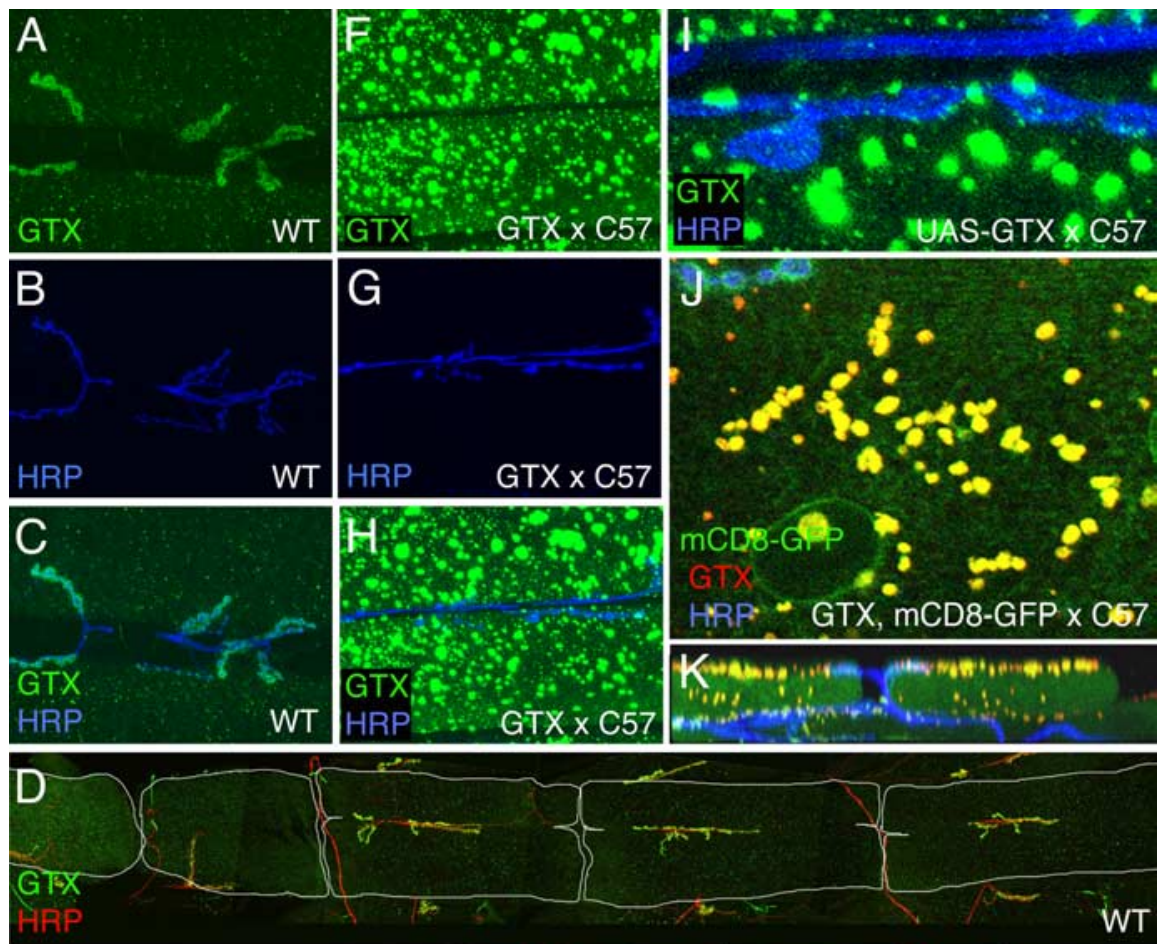

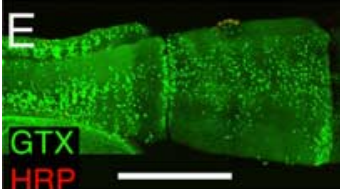

A1

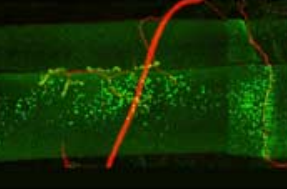

A2

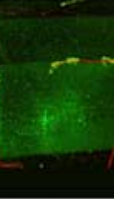

$\mathrm{A} 3$

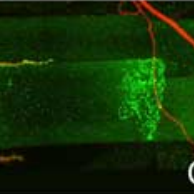

3
Figure 7. Effects of overexpressing GTX in larval muscles. $\boldsymbol{A}-\boldsymbol{K}$, Confocal images of larval NMJs in wild type $(\boldsymbol{A}-\boldsymbol{D})$ and larvae overexpressing transgenic GTX (E- $\boldsymbol{K})$ in muscles using the Gal4 driver BG487 (D, $\boldsymbol{E})$ (weak muscle expression in $\boldsymbol{A 3}$ ) and $(57(\boldsymbol{F}-\boldsymbol{K})$ (strong pan-muscle expression), stained with antibodies to HRP (blue) and GTX (green) ( $\boldsymbol{A}-\boldsymbol{C}, \boldsymbol{F}-\boldsymbol{I}), \mathrm{GTX}$ (green) and HRP (red) (D. $\boldsymbol{E}$ ), and GTX (red), HRP (blue), and mCD8-GFP (green) $(\boldsymbol{J}, \boldsymbol{K}) . \boldsymbol{A}-\boldsymbol{C}$ and $\boldsymbol{F}$ - $\boldsymbol{K}$ are images from muscles 6 and 7 in $\boldsymbol{A} \mathbf{3}$, whereas $\boldsymbol{D}$ and $\boldsymbol{E}$ show abdominal segments $\boldsymbol{A 1} \mathbf{A} \boldsymbol{A}$. $\boldsymbol{K}$ is a transversal section through a confocal stack showing the localization of GTX-containing large vesicles at the muscle apical region. Scale bar: $\boldsymbol{A}-\boldsymbol{C}, \boldsymbol{F}-\boldsymbol{H}, 50 \mu \mathrm{m} ; \boldsymbol{D}, \boldsymbol{E}, 185 \mu \mathrm{m} ; \boldsymbol{I}, 8 \mu \mathrm{m} ; \boldsymbol{J}, 12 \mu \mathrm{m} ; \boldsymbol{K}, 30 \mu \mathrm{m}$.

(Guan et al., 1996). How is this process specifically targeted to synaptic sites, which correspond to $<1 \%$ of the muscle surface? Our finding that DLG interacts with and regulates GTX provides new insight on this process. GTX binds the GUK domain of DLG, and the postsynaptic localization of GTX depends on DLG. Most notably, gtx mutants exhibit a dramatic reduction in SSR length, and a GTX-containing SNARE complex was decreased in $d l g$ mutants and enhanced when DLG is overexpressed. We propose that DLG directs membrane addition to the SSR by regulating the targeting and activity of GTX (Fig. 9C).

Several lines of evidence support this model. First, enrichment of GTX at the SSR is strongly dependent on the presence and correct localization of DLG. When DLG is moderately overexpressed, the localization of GTX at the SSR is enhanced. Moreover, strongly overexpressed DLG leads to increased association of DLG with intramuscular compartments, which caused GTX to accumulate extrasynaptically. This DLG dose dependency of GTX targeting provides compelling evidence that DLG acts as a strong determinant of GTX localization. Second, mutations in $g t x$, like mutations in $d l g$, lead to an underdeveloped SSR, suggesting that GTX and DLG are in the same membrane addition pathway. Third, overexpressing GTX leads to loss of SSR at sites of synaptic contact and a DLG-independent formation of ectopic
GTX x BG487 A4
SSR-like structures. These ectopic structures were still present when GTX was overexpressed in $d l g$ mutants, suggesting that GTX-mediated fusion can occur in the absence of DLG when GTX concentration is very high. These observations suggest that GTX plays a role in fusion of membranes to form the SSR. Interestingly, the ectopic SSR-like structures did not appear to contain any of the postsynaptic proteins found within the SSR, including DLG, Scribble, Fasciclin II, and GluRs. Therefore, trafficking of SSR proteins is somewhat independent from the process of membrane addition to the SSR. Fourth, low GTX expression, could rescue the gtx mutant SSR defects, but not those of $d l g$ mutants, further supporting the model that GTX requires DLG for membrane addition to the SSR.

The role of DLG in SSR formation may extend beyond simply recruiting GTX to the SSR. We propose a model that further explains how molecular interactions between DLG and GTX may direct SSR formation (Fig. 9D). First, the immunoprecipitation studies suggest that DLG associates more strongly with higher molecular weight complexes containing GTX, rather than with monomeric GTX. We propose that the low-affinity interaction between monomeric GTX and DLG directs recruitment of GTX to the SSR. Once at the SSR, homotypic interaction between GTX monomers leads to the formation of GTX SNARE complexes. Second, at least one of the GTX SNARE complexes $(70 \mathrm{kDa})$ was decreased in $d l g$ mutants and increased by DLG overexpression. This complex could not be restored by overexpressing GTX in a dlg mutant. Therefore, the high-affinity interaction between DLG and GTX SNARE complexes may lead to their protection/stabilization, thereby facilitating membrane fusion precisely at sites of synaptic contact.

GTX is the putative homolog of Syntaxin-18 and Ufe1p. Unlike other t-SNARES, these proteins can mediate homotypic membrane fusion in the absence of other known SNAREs (Patel et al., 1998; Kano et al., 2005). Thus, targeting GTX-containing vesicles to specific membrane compartments would result in membrane expansion. Consistently, Syntaxin-18 and Ufe1p have been implicated in mediating vesicle fusion as vesicles traffic from one ER compartment to another (Lewis and Pelham, 1996; Hatsuzawa et al., 2000). The presumptive role of GTX as part of an ER-specific vesicle fusion machinery, together with its requirement for SSR development, supports the idea that the SSR bears at least some ER-like properties. Moreover, the role of a so-called ER to Golgi SNARE in a specialized plasma membrane such as the SSR is not surprising, because Syntaxin-18 has also been implicated recently in the function of the phagosome during immunoglobulin-mediated particle engulfment by professional phagocytes (Hatsuzawa et al., 2006).

Both DLG and GTX are exclusively localized at type I terminals and not in other terminals such as type II or type III, which 
lack SSR (Jia et al., 1993). Some muscle fibers such as muscle 12 are innervated by all three types of terminals (Gorczyca et al., 1993; Jia et al., 1993), suggesting that in the same postsynaptic cell, there are selective membrane-trafficking systems in place.

Mutations in gtx also resulted in electrophysiological changes. The decrease in mEJP frequency is consistent with the finding that $g t x$ mutants have reduced number of boutons, without affecting the number of active zones per bouton. Another very interesting phenotype was the decrease in EJP decay constant. This was not a result of changes in passive properties or to changes in GluR function. It is quite possible that the trafficking of a voltage gated channel is altered in $g t x$ mutants. For example, an increase in the function of a $\mathrm{K}^{+}$channel would be expected to generate a similar change in EJP kinetics.

\section{Dual function of GTX in synaptic expansion and muscle growth} We demonstrated that GTX also plays a role during muscle growth. Notably, the synaptic and muscle functions could be genetically uncoupled. Whereas muscle size was primarily insensitive to elevated GTX levels, a fairly precise dosage of GTX is required for synaptic growth. DLG itself may also play a role during muscle membrane trafficking, in addition to its more notable role in synapse development. Despite the enrichment of DLG at synaptic boutons, it is also present in the muscle, where it intermingles with the myocontractile apparatus, in the subcortical network, and in association with T-tubules (Razzaq et al., 2001). It is thus possible that DLG also operates during the localization of proteins required for excitationcontraction coupling.

The analysis of $d l g$ and $g t x$ mutants using mCD8-GFP to label membranes has also uncovered a role of GTX in the morphogenesis of the cortical membrane compartment. Previous studies have shown that DLG traffics through this compartment on its way to postsynaptic sites (Thomas et al., 2000). In gtx mutants, the cortical network appears to have collapsed into the subcortical network, which may explain the slight defects in DLG trafficking observed in gtx mutants.

\section{Significance in other systems}

Living cells are highly specialized machines that must filter a vast array of information from the environment to integrate into sophisticated higher-order networks such as organs and neural systems. Cellular signaling is organized into specialized networks of proteins, the function of which is often structured by the modular protein-protein interaction domains of scaffolding proteins. Al-

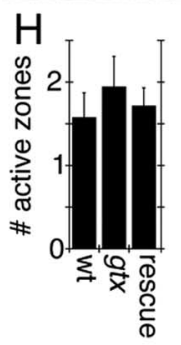

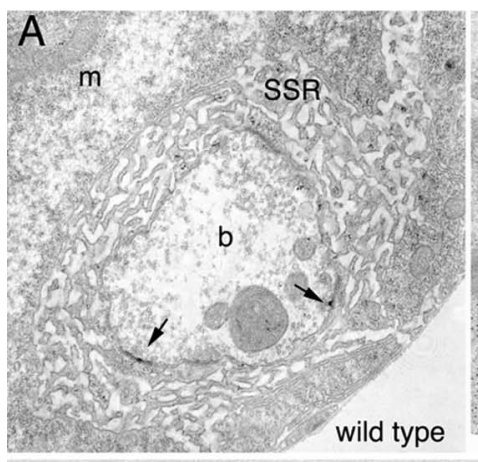
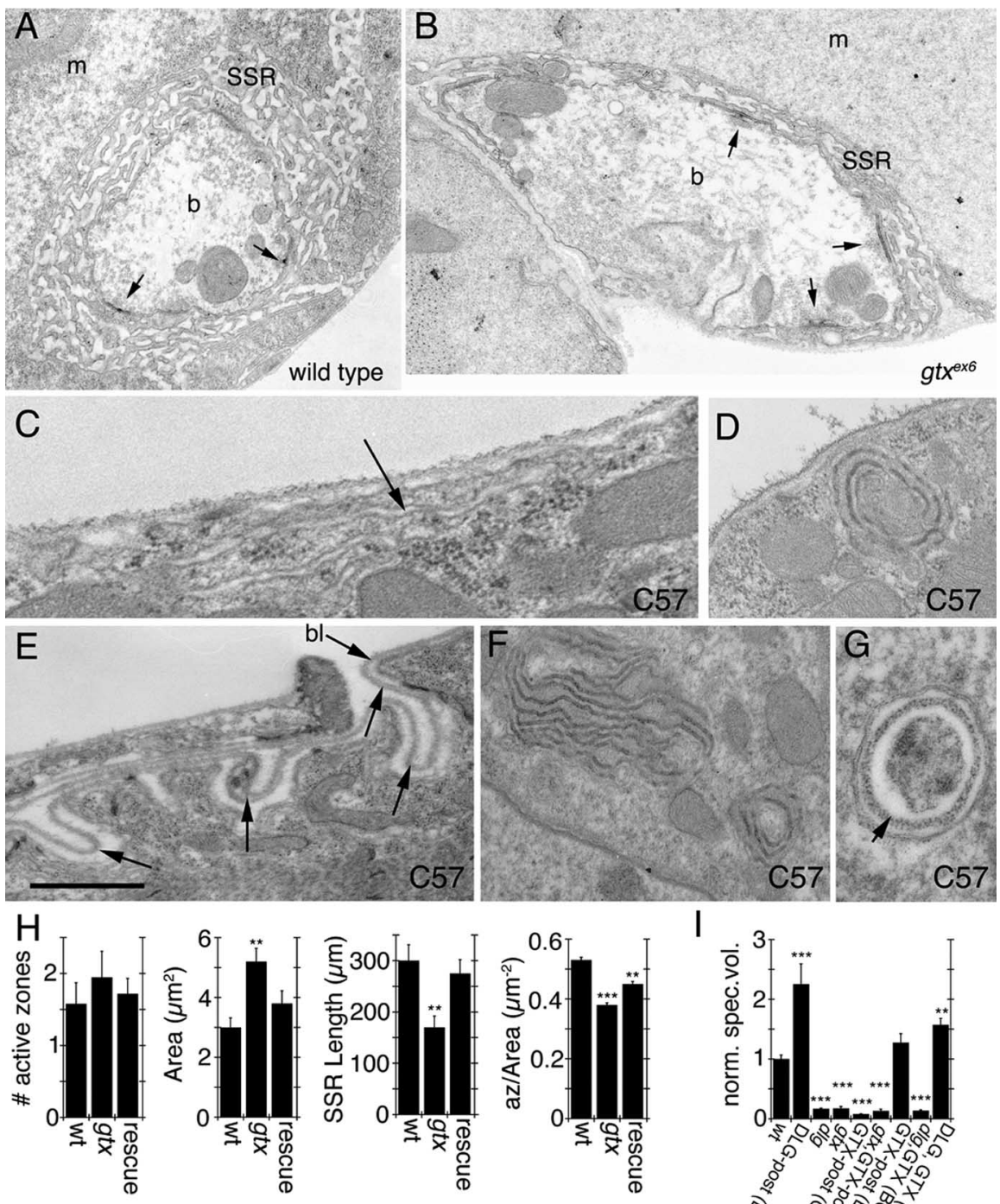

Figure 8. Ultrastructure of synaptic boutons and muscles in gtx mutants. $\boldsymbol{A}-\boldsymbol{G}$, Electron micrographs of type lb synaptic boutons $(\boldsymbol{A}, \boldsymbol{B})$ in wild type $(\boldsymbol{A})$ and $g t x^{e x 6}$ mutants $(\boldsymbol{B})$. $m$, Muscle; $b$, bouton. Arrows point to T-bar active zones. $\boldsymbol{C}-\boldsymbol{G}$, Extrasynaptic membrane structures observed in larvae overexpressing GTX using the strong pan-muscle Gal 4 driver $C 57$. Arrows in $\boldsymbol{C}$ and $\boldsymbol{E}$ point to surface membranous structures resembling the SSR, and the arrow in $\mathbf{G}$ points to electron dense material resembling basal lamina (bl). $\boldsymbol{H}$, Morphometric analysis of synaptic boutons in wild type, $g t x^{e x 6}$ mutants, and $g t x^{e x 6}$ mutants expressing transgenic GTX using the Gal4 strain BG487. Numbers analyzed were 12 boutons in two wild-type larvae, 19 boutons in two gtx ${ }^{\text {ex6 }}$, and 12 boutons in three $g t{ }^{e x 6}$ expressing GTX in muscles using the BG487 Gal4. I, Quantification of the volume occupied by Spectrin at the postsynaptic area in different genotypes. $n=6$ samples for each phenotype; ${ }^{* *} p=0.001$; ${ }^{* * *} p<0.0001 . n=6$ for $g t x^{e x 6}$ and $g t x^{e x 6} ;$ C57 and 8 for all other genotypes. Scale bar: $\boldsymbol{A}, \boldsymbol{B}, 1.5 \mu \mathrm{m} ; \boldsymbol{C}, 0.2 \mu \mathrm{m} ; \boldsymbol{D}, \boldsymbol{F}, 0.5 \mu \mathrm{m} ; \boldsymbol{E}, \boldsymbol{G}, 0.3$.

though much has been learned about the role of scaffolding proteins in the compartmentalization of polarized signaling complexes and the physical linkage of cell adhesion molecules to the cytoskeleton (Kim and Sheng, 2004; Funke et al., 2005), there have been few examples of how scaffolding proteins may also guide other processes such as vesicle fusion to specific cellular domains.

In mammals, the development and activation state of specific synapses during plasticity is accompanied by dramatic changes in the size of postsynaptic dendritic spines and the ER within spines (Marrs et al., 2001; Nimchinsky et al., 2002; Oray et al., 2004; Cathala et al., 2005; Toresson and Grant, 2005). It is likely that 


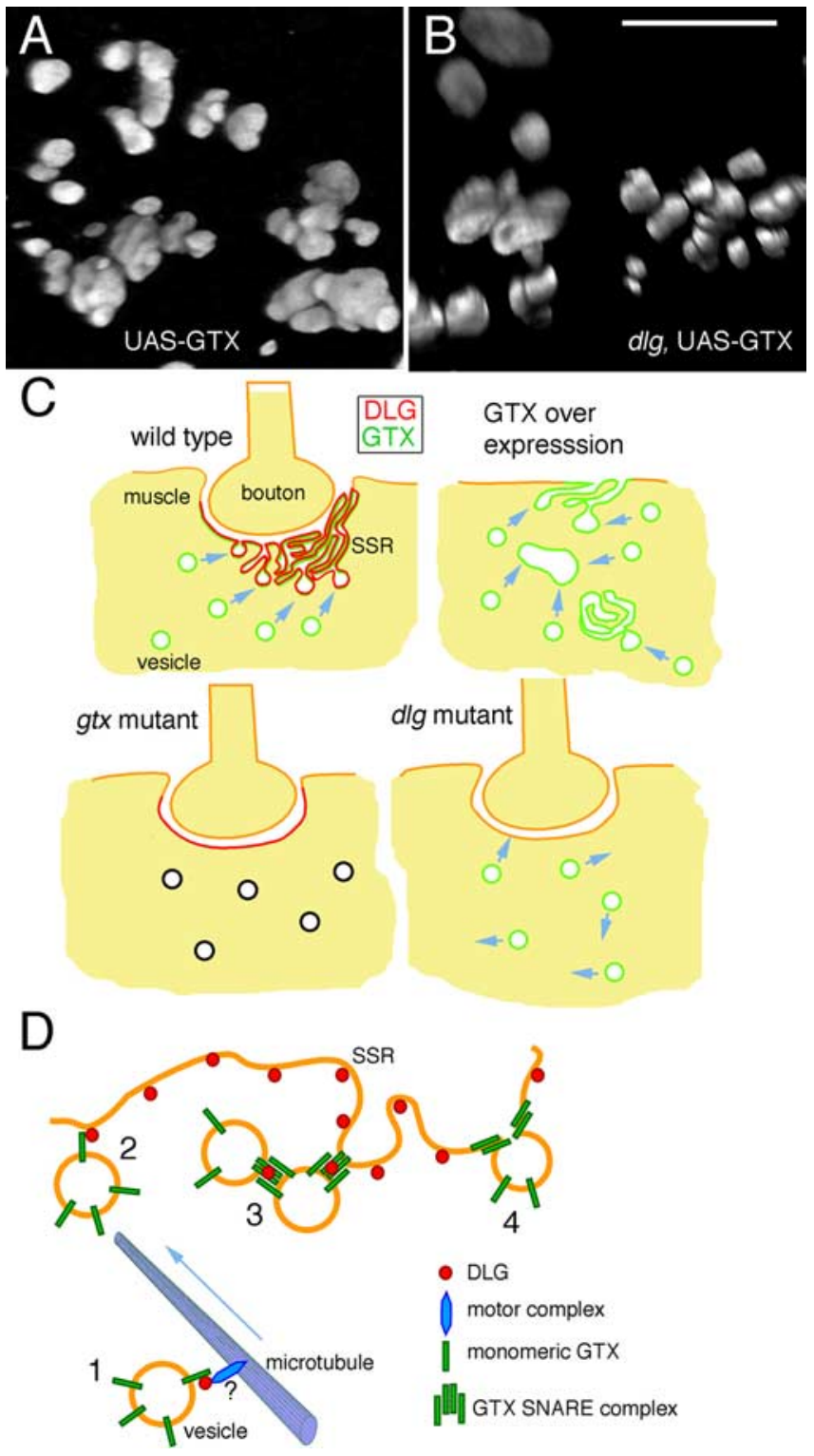

Figure 9. Ectopic SSR formation after strong GTX overexpression and proposed function of DLG and GTX during targeted membrane addition. $\boldsymbol{A}, \boldsymbol{B}$, Three-dimensional renderings of ectopic GTX-positive structures observed after overexpressing GTX with the strong $C 57$ driver in wild type $(\boldsymbol{A})$ and $d \mathrm{ll} g^{X I-2}$ mutant $(\boldsymbol{B})$ backgrounds. Scale bar: $\boldsymbol{A}, \boldsymbol{B}, 8 \mu \mathrm{m}$. C, Model of membrane trafficking to the postsynaptic region. In wild type, the presence of DLG at the postsynaptic membrane directs the fusion of GTX-positive vesicles to this site, forming the SSR. In dlg mutants, GTX targeting to the postsynaptic membrane is perturbed, resulting in reduced SSR expansion. When GTX is severely reduced in gtx mutants, despite the relatively normal synaptic DLG localization, fusion events are inhibited, and the SSR does not expand. When GTX is expressed at very high levels, inappropriate homotypic fusion occurs, leading to the formation of large vesicles and SSR-like structures in the cytoplasm and extrasynaptic muscle surface. $\boldsymbol{D}$, Potential interactions between DLG and GTX during SSR formation. 1, GTX-positive vesicles are trafficked to the SSR, perhaps in a DLG dependent manner. 2, Through low-affinity interactions between DLG and GTX monomers, GTX-containing vesicles are concentrated at the SSR region. 3, Increased concentration of GTX-containing vesicles facilitates the formation of SNARE complexes, which are stabilized/protected by DLG. 4, The GTX SNARE complex formations allow the fusion of GTX vesicles with the SSR membrane, thus resulting in SSR expansion.

membrane addition events such as the ones described here also occur in a targeted manner, and this process may also be regulated by MAGUKs (Riefler et al., 2003). Indeed, changes in PSD-95 levels elicit striking changes in spine size (El-Husseini et al., 2000a). It is possible that this may also occur though regulation of SNAREs such as GTX.

\section{References}

Ashley J, Packard M, Ataman B, Budnik V (2005) Fasciclin II signals new synapse formation through amyloid precursor protein and the scaffolding protein dX11/Mint. J Neurosci 25:5943-5955.

Budnik V, Zhong Y, Wu CF (1990) Morphological plasticity of motor axons in Drosophila mutants with altered excitability. J Neurosci 10:3754-3768. Budnik V, Koh YH, Guan B, Hartmann B, Hough C, Woods D, Gorczyca M (1996) Regulation of synapse structure and function by the Drosophila tumor suppressor gene dlg. Neuron 17:627-640.

Cathala L, Holderith NB, Nusser Z, DiGregorio DA, Cull-Candy SG (2005) Changes in synaptic structure underlie the developmental speeding of AMPA receptor-mediated EPSCs. Nat Neurosci 8:1310-1318.

El-Husseini AE, Schnell E, Chetkovich DM, Nicoll RA, Bredt DS (2000a) PSD-95 involvement in maturation of excitatory synapses. Science 290:1364-1368.

El-Husseini AE, Craven SE, Chetkovich DM, Firestein BL, Schnell E, Aoki C, Bredt DS (2000b) Dual palmitoylation of PSD-95 mediates its vesiculotubular sorting, postsynaptic targeting, and ion channel clustering. J Cell Biol 148:159-172.

Funke L, Dakoji S, Bredt DS (2005) Membrane-associated guanylate kinases regulate adhesion and plasticity at cell junctions. Annu Rev Biochem 74:219-245.

Gorczyca M, Augart C, Budnik V (1993) Insulin-like receptor and insulinlike peptide are localized at neuromuscular junctions in Drosophila. J Neurosci 13:3692-3704.

Guan B, Hartmann B, Kho YH, Gorczyca M, Budnik V (1996) The Drosophila tumor suppressor gene, dlg, is involved in structural plasticity at a glutamatergic synapse. Curr Biol 6:695-706.

Hatsuzawa K, Hirose H, Tani K, Yamamoto A, Scheller RH, Tagaya M (2000) Syntaxin 18, a SNAP receptor that functions in the endoplasmic reticulum, intermediate compartment, and cis-Golgi vesicle trafficking. J Biol Chem 275:13713-13720.

Hatsuzawa K, Tamura T, Hashimoto H, Hashimoto H, Yokoya S, Miura M, Nagaya H, Wada I (2006) Involvement of syntaxin 18, an endoplasmic reticulum (ER)-localized SNARE protein, in ER-mediated phagocytosis. Mol Biol Cell 21:21.

Hong W (2005) SNAREs and traffic. Biochim Biophys Acta 1744:493-517.

Horton AC, Ehlers MD (2004) Secretory trafficking in neuronal dendrites. Nat Cell Biol 6:585-591.

Hung AY, Sheng M (2001) PDZ domains: structural modules for protein complex assembly. J Biol Chem 277:5699-5702.

Jia XX, Gorczyca M, Budnik V (1993) Ultrastructure of neuromuscular junctions in Drosophila: comparison of wild type and mutants with increased excitability. J Neurobiol [Erratum (1994) 25:893-895] 24:1025-1044.

Kano F, Kondo H, Yamamoto A, Kaneko Y, Uchiyama K, Hosokawa N, Nagata K, Murata M (2005) NSF/SNAPs and p97/p47/VCIP135 are sequentially required for cell cycle-dependent reformation of the ER network. Genes Cells 10:989-999.

Kim E, Sheng M (2004) PDZ domain proteins of synapses. Nat Rev Neurosci 5:771-781.

Koh YH, Popova E, Thomas U, Griffith LC, Budnik V (1999) Regulation of DLG localization at synapses by CaMKII-dependent phosphorylation. Cell 98:353-363.

Lahey T, Gorczyca M, Jia XX, Budnik V (1994) The Drosophila tumor suppressor gene dlg is required for normal synaptic bouton structure. Neuron 13:823-835.

Lee WC, Huang H, Feng G, Sanes JR, Brown EN, So PT, Nedivi E (2006) Dynamic remodeling of dendritic arbors in GABAergic interneurons of adult visual cortex. PLoS Biol 4:e29.

Lewis MJ, Pelham HR (1996) SNARE-mediated retrograde traffic from the Golgi complex to the endoplasmic reticulum. Cell 85:205-215.

Marrs GS, Green SH, Dailey ME (2001) Rapid formation and remodeling of postsynaptic densities in developing dendrites. Nat Neurosci 4:1006-1013.

Mathew D, Gramates LS, Packard M, Thomas U, Bilder D, Perrimon N, Gorczyca M, Budnik V (2002) Recruitment of scribble to the synaptic scaffolding complex requires GUK-holder, a novel DLG binding protein. Curr Biol 12:531-539.

Mathew D, Popescu A, Budnik V (2003) Drosophila amphiphysin functions during synaptic Fasciclin II membrane cycling. J Neurosci 23:10710-10716. 
Mathew D, Ataman B, Chen J, Zhang Y, Cumberledge S, Budnik V (2005) Wingless signaling at synapses is through cleavage and nuclear import of receptor DFrizzled2. Science 310:1344-1347.

Matsuzaki M, Honkura N, Ellis-Davies GC, Kasai H (2004) Structural basis of long-term potentiation in single dendritic spines. Nature 429:761-766.

Mendoza C, Olguin P, Lafferte G, Thomas U, Ebitsch S, Gundelfinger ED, Kukuljan M, Sierralta J (2003) Novel isoforms of Dlg are fundamental for neuronal development in Drosophila. J Neurosci 23:2093-2101.

Monastirioti M, Gorczyca M, Rapus J, Eckert M, White K, Budnik V (1995) Octopamine immunoreactivity in the fruit fly Drosophila melanogaster. J Comp Neurol 356:275-287.

Nimchinsky EA, Sabatini BL, Svoboda K (2002) Structure and function of dendritic spines. Annu Rev Physiol 64:313-353.

Nourry C, Grant SG, Borg JP (2003) PDZ domain proteins: plug and play! Sci STKE 2003:RE7.

Oray S, Majewska A, Sur M (2004) Dendritic spine dynamics are regulated by monocular deprivation and extracellular matrix degradation. Neuron 44:1021-1030.

Otto H, Hanson PI, Jahn R (1997) Assembly and disassembly of a ternary complex of synaptobrevin, syntaxin, and SNAP-25 in the membrane of synaptic vesicles. Proc Natl Acad Sci USA 94:6197-6201.

Packard M, Koo ES, Gorczyca M, Sharpe J, Cumberledge S, Budnik V (2002) The Drosophila wnt, wingless, provides an essential signal for pre- and postsynaptic differentiation. Cell 111:319-330.

Pak DT, Yang S, Rudolph-Correia S, Kim E, Sheng M (2001) Regulation of dendritic spine morphology by SPAR, a PSD-95-associated RapGAP. Neuron 31:289-303.

Patel SK, Indig FE, Olivieri N, Levine ND, Latterich M (1998) Organelle membrane fusion: a novel function for the syntaxin homolog Ufelp in ER membrane fusion. Cell 92:611-620.

Petersen SA, Fetter RD, Noordermeer JN, Goodman CS, DiAntonio A (1997) Genetic analysis of glutamate receptors in Drosophila reveals a retrograde signal regulating presynaptic transmitter release. Neuron 19:1237-1248.

Razzaq A, Robinson IM, McMahon HT, Skepper JN, Su Y, Zelhof AC, Jackson AP, Gay NJ, O’Kane CJ (2001) Amphiphysin is necessary for organization of the excitation-contraction coupling machinery of muscles, but not for synaptic vesicle endocytosis in Drosophila. Genes Dev 15:2967-2979.

Riefler GM, Balasingam G, Lucas KG, Wang S, Hsu SC, Firestein BL (2003) Exocyst complex subunit sec8 binds to postsynaptic density protein-95 (PSD-95): a novel interaction regulated by cypin (cytosolic PSD-95 interactor). Biochem J 373 49-55.

Roche JP, Packard MC, Moeckel-Cole S, Budnik V (2002) Regulation of synaptic plasticity and synaptic vesicle dynamics by the PDZ protein Scribble. J Neurosci 22:6471-6479.

Rorth P (1996) A modular misexpression screen in Drosophila detecting tissue-specific phenotypes. Proc Natl Acad Sci USA 93:12418-12422.

Ruiz-Canada C, Ashley J, Moeckel-Cole S, Drier E, Yin J, Budnik V (2004) New synaptic bouton formation is disrupted by misregulation of microtubule stability in aPKC mutants. Neuron 42:567-580.
Sans N, Racca C, Petralia RS, Wang YX, McCallum J, Wenthold RJ (2001) Synapse-associated protein 97 selectively associates with a subset of AMPA receptors early in their biosynthetic pathway. J Neurosci 21:7506-7516.

Schuster CM, Davis GW, Fetter RD, Goodman CS (1996a) Genetic dissection of structural and functional components of synaptic plasticity. II. Fasciclin II controls presynaptic structural plasticity. Neuron 17:655-667.

Schuster CM, Davis GW, Fetter RD, Goodman CS (1996b) Genetic dissection of structural and functional components of synaptic plasticity. I. Fasciclin II controls synaptic stabilization and growth. Neuron 17:641-654.

Sigrist SJ, Thiel PR, Reiff DF, Lachance PE, Lasko P, Schuster CM (2000) Postsynaptic translation affects the efficacy and morphology of neuromuscular junctions. Nature 405:1062-1065.

Sollner T, Whiteheart SW, Brunner M, Erdjument-Bromage H, Geromanos S, Tempst P, Rothman JE (1993) SNAP receptors implicated in vesicle targeting and fusion. Nature 362:318-324.

Sone M, Suzuki E, Hoshino M, Hou D, Kuromi H, Fukata M, Kuroda S, Kaibuchi K, Nabeshima Y, Hama C (2000) Synaptic development is controlled in the periactive zones of Drosophila synapses. Development 127:4157-4168.

Stewart BA, Atwood HL, Renger JJ, Wang J, Wu CF (1994) Improved stability of Drosophila larval neuromuscular preparations in haemolymphlike physiological solutions. J Comp Physiol A Neuroethol Sens Neural Behav Physiol 175:179-191.

Tejedor FJ, Bokhari A, Rogero O, Gorczyca M, Zhang J, Kim E, Sheng M, Budnik V (1997) Essential role for dlg in synaptic clustering of Shaker $\mathrm{K}^{+}$channels in vivo. J Neurosci 17:152-159.

Thomas U, Kim E, Kuhlendahl S, Koh YH, Gundelfinger ED, Sheng M, Garner CC, Budnik V (1997) Synaptic clustering of the cell adhesion molecule fasciclin II by discs-large and its role in the regulation of presynaptic structure. Neuron 19:787-799.

Thomas U, Ebitsch S, Gorczyca M, Koh YH, Hough CD, Woods D, Gundelfinger ED, Budnik V (2000) Synaptic targeting and localization of discs-large is a stepwise process controlled by different domains of the protein. Curr Biol 10:1108-1117.

Toresson H, Grant SG (2005) Dynamic distribution of endoplasmic reticulum in hippocampal neuron dendritic spines. Eur J Neurosci 22:1793-1798.

Woods DF, Bryant PJ (1989) Molecular cloning of the lethal(1)discs large-1 oncogene of Drosophila. Dev Biol 134:222-235.

Woods DF, Hough C, Peel D, Callaini G, Bryant PJ (1996) Dlg protein is required for junction structure, cell polarity, and proliferation control in Drosophila epithelia. J Cell Biol 134:1469-1482.

Zito K, Fetter RD, Goodman CS, Isacoff EY (1997) Synaptic clustering of Fascilin II and Shaker: essential targeting sequences and role of Dlg. Neuron 19:1007-1016. 